\title{
Mycobacterial brain tuberculomas due to Bacille Calmette-Guérin intravesical chemotherapy for bladder cancer: A case report and literature review
}

\author{
Vitaly Golub MD, Prashant Malhotra MD, Shital Patel MD
}

\begin{abstract}
V Golub, P Malhotra, S Patel. Mycobacterial brain tuberculomas due to Bacille Calmette-Guérin intravesical chemotherapy for bladder cancer: A case report and literature review. Can J Infect Dis Med Microbiol 2011;22(3):104-106.

Bacille Calmette-Guérin (BCG) immunotherapy is widely used for the treatment of superficial bladder cancer. The authors believe that the present report is one of the first to document cerebral BCG tuberculoma in a 73-year-old immunocompetent man, three years after intravesical BCG immunotherapy. His workup revealed no identifiable extracranial source. He responded well to treatment with rifampin, ethambutol and moxifloxacin.

Patients undergoing intravesical BCG therapy should be closely monitored for the development of this complication. Prolonged antitubercular therapy, possibly including moxifloxacin, appears to be beneficial in the treatment of central nervous system tuberculous infections.
\end{abstract}

Key Words: BCG; Bladder cancer; Cerebral; Tuberculoma

\author{
Des tuberculomes cérébraux mycobactériens \\ causés par une chimiothérapie intravésicale par \\ le bacille de Calmette-Guérin pour le \\ traitement d'un cancer de la vessie : un rapport \\ de cas et une analyse bibliographique
}

L'immunothérapie par le bacille de Calmette-Guérin (BCG) est largement utilisée pour traiter le cancer superficiel de la vessie. Les auteurs pensent que le présent cas est l'un des premiers à rendre compte d'un tuberculome cérébral par le BCG chez un homme immunocompétent de 73 ans, trois ans après une immunothérapie intravésicale par le BCG. Son bilan n'a révélé aucune source extracrânienne repérable. Il a bien réagi au traitement à la rifampine, à l'éthambutol et à la moxifloxacine. Les patients qui subissent une thérapie intravésicale par le BCG devraient faire l'objet d'un suivi étroit pour déceler l'apparition de cette complication. Une thérapie antituberculaire prolongée, pouvant inclure de la moxifloxacine, semble bénéfique pour le traitement des infections tuberculeuses du système nerveux central.
Racille Calmette-Guérin (BCG), a live attenuated strain of wildDtype Mycobacterium bovis, is widely used for vaccination against tuberculosis. Since 1976, it has been shown to be highly effective in the treatment of superficial bladder carcinoma (1). Treatment of the latter appears to be relatively safe, with major adverse reactions occurring in fewer than $5 \%$ of patients (2). Documented complications of BCG immunotherapy include granulomatous pneumonitis, hepatitis, prostatitis, vascular aneurysm infections and vertebral osteomyelitis (3).

Central nervous system (CNS) infections caused by M bovis are rare, and those caused by BCG are described in only a few case reports (4-6). CNS infections due to Mycobacterium tuberculosis range from meningitis to tuberculomas, which are tumour-like masses with occasional central necrosis. We describe what we believe is one of the first documented cases of cerebral BCG tuberculoma observed in a 73-year-old immunocompetent man, three years after he underwent intravesical BCG immunotherapy for transitional cell carcinoma of the bladder.

\section{CASE PRESENTATION}

A 73-year-old man presented to the North Shore University Hospital (New York, USA) in January 2009 with a three-week history of headaches, dizziness and right hand tremor. The patient denied fevers, chills, neck pain or stiffness, confusion or cough. He denied night sweats and weight loss. His medical history was significant for prostate cancer treated with radioactive seeds, and transitional bladder carcinoma treated with serial intravesical BCG injections at weekly intervals for six weeks. His most recent BCG cycle occurred approximately three years before admission. He experienced no adverse reactions during treatment. The patient was not taking corticosteroids or any other medications at the time of presentation. He was born in the United States and denied travel outside of the country, exposure to tuberculosis, alcohol, tobacco or injection drug use. His purified protein derivative/ Mantoux testing status was unknown.

On physical examination, the patient was afebrile with normal vital signs. His neurological examination was significant only for a right hand resting tremor. Routine laboratory values including complete blood count, hepatic panel and a renal panel were unremarkable. The initial computed tomography scan of his brain (without intravenous contrast) failed to reveal any pathology; the patient subsequently underwent magnetic resonance imaging (MRI) of the brain. The MRI (Figure 1) showed three patchy and nodular foci of enhancement within the left frontal, temporal and basal ganglia regions. Computed tomography scans of the neck, chest, abdomen and pelvis were unremarkable. The patient subsequently underwent a stereotactic biopsy of the lesion. The pathology was consistent with chronic granulomatous inflammation. The tissue samples were acidfast bacilli (AFB) smear negative. A cerebrospinal fluid (CSF) analysis was also performed and revealed 7 cells $/ \mu \mathrm{L}$ (normal 0 cells $/ \mu \mathrm{L}$ to 5 cells $/ \mu \mathrm{L}$ ) with $61 \%$ lymphocytes, CSF glucose $77 \mathrm{mg} / \mathrm{dL}$ (normal range $50 \mathrm{mg} / \mathrm{dL}$ to $80 \mathrm{mg} / \mathrm{dL}$ ) and CSF protein $85 \mathrm{mg} / \mathrm{dL}$ (normal range $15 \mathrm{mg} / \mathrm{dL}$ to $60 \mathrm{mg} / \mathrm{dL}$ ). The patient's purified protein derivative/Mantoux test (performed during hospitalization) and an HIV test were negative.

The patient was initiated on a four-drug antitubercular regimen (ie, isoniazid, rifampin, ethambutol and pyrazinamide) for presumed cerebral tuberculomas. After four weeks, growth of dysgonic colonies was observed on Lowenstein-Jensen media. Polymerase chain reaction assays of the tissue identified $M$ tuberculosis complex ribosomal RNA using the AccuProbe Mycobacterium tuberculosis Complex Culture Identification Kit (Gen-Probe Inc, USA). His work up including sputum AFB smear and culture, CSF bacterial, fungal and AFB cultures, as well as brain tissue bacterial and fungal cultures, 
remained negative at six weeks. The patient was continued on the above four medications.

Six weeks later, the New York State Department of Health Mycobacteriology laboratory (Wadsworth Center, USA) using an inhouse polymerase chain reaction-based genomic deletion analysis (7) for regions of difference (RD) 1, RD9 and RD 10, identified the isolate as an $\mathrm{M}$ bovis BCG strain. Sensitivity testing using the Bactec MGIT 960 system (Becton Dickinson, USA) indicated that the strain was resistant to isoniazid (at a concentration of $0.1 \mu \mathrm{g} / \mathrm{mL}$ ) and pyrazinamide (at a concentration of $100 \mu \mathrm{g} / \mathrm{mL}$ ), but sensitive to rifampin, ethambutol and ciprofloxacin. At this time, isoniazid and pyrazinamide were discontinued and moxifloxacin was started.

One year later, the patient is doing well on ethambutol, moxifloxacin and rifampin. Serial MRIs of the brain have shown a decrease in the size of the cerebral lesions. His right hand tremor has resolved.

\section{DISCUSSION}

$\mathrm{M}$ bovis, the agent responsible for bovine tuberculosis, is also known to cause zoonotic infections. $M$ bovis is a member of the $M$ tuberculosis complex, which includes $M$ tuberculosis, Mycobacterium africanum and Mycobacterium microti. While similar to $\mathrm{M}$ tuberculosis, $\mathrm{M}$ bovis is uniformly resistant to pyrazinamide, and has a different composition on a nucleic acid probe. Most human exposure stems from raw milk ingestion and inhalation of contaminated droplets (8). In the current era, $\mathrm{M}$ bovis constitutes a fraction of human tuberculosis cases. In a 1995 survey from France (8), $0.5 \%$ of human tuberculosis cases and, more recently, $1.4 \%$ of United States human tuberculosis cases reported (9) were due to $M$ bovis. The vast majority of the patients in the latter study were of Mexican descent. Pulmonary and extrapulmonary manifestations of $\mathrm{M}$ bovis are described in the literature. However, the digestive route of acquisition has been largely eliminated with milk pasteurization.

BCG is an attenuated form of wild-type M bovis and has been used for vaccination against human tuberculosis. Since 1976, it has also been used for the treatment of superficial bladder cancer, and approved for the treatment of carcinoma in situ (2). It is believed to exert its antitumour activity via local modulation of immune responses, which results in inflammation and subsequent elimination of malignant cells (10). The most widely used regimen consists of a weekly intravesical BCG injection for six weeks, which is repeated three more times every six months (2).

While generally well tolerated, complications with BCG immunotherapy have been well documented. Most of the early manifestations of BCG disease are characterized by a hypersensitivity type of reaction, namely, pneumonitis, hepatitis or, rarely, sepsis. Fever, chills, sweats and malaise within three months of instillation are the most common presenting symptoms (3). In a Cochrane database review (11) comparing transurethral resection of the tumour with BCG immunotherapy, the most commonly reported complications were fever (25\%), cystitis $(67 \%)$ and hematuria (23\%). While the latter reported no deaths due to BCG therapy, fatal sepsis is described in the literature at an approximate rate of one per 15,000 patients (2).

There are no standard guidelines for the treatment of BCG infections occurring as a complication of intravesical therapy. Although most of the early manifestations are believed to be secondary to hypersensitivity, some authors recommend treatment with isoniazid or fluoroquinolone if symptoms persist beyond $48 \mathrm{~h}$ (12). In addition, a tapered course of prednisone is occasionally used for hypersensitivity.

Late infections with BCG are characterized by a spectrum of manifestations. In contrast to early disease, which frequently affects more than one organ, late disease is usually localized. Moreover, late manifestation disease is more likely to yield a positive culture. In a review (11) of BCG infections after bladder cancer treatment, the predominant manifestations were testicular and mycotic aneurysm granulomas. Common to both early and late disease is the pathological diagnosis of a non-necrotizing granuloma. Disseminated or severe infections usually require a prolonged course (ie, six to 12 months) usually with a combination of two or three drugs.

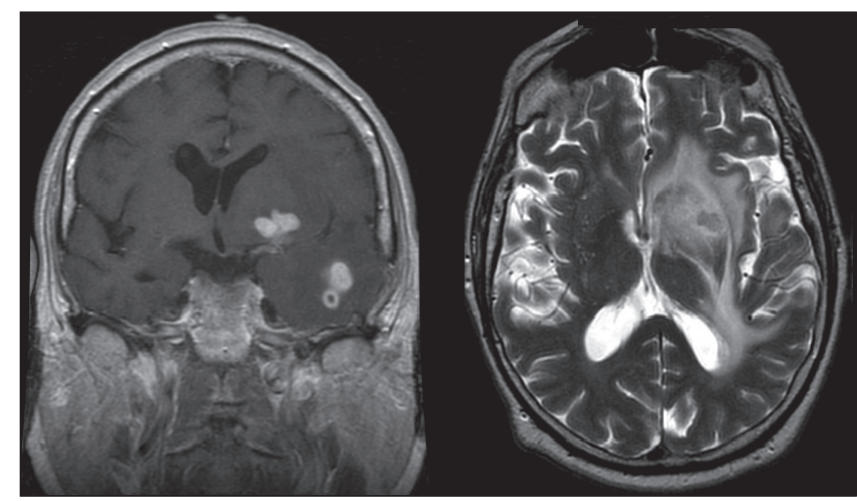

Figure 1) Postcontrast T1 coronal magnetic resonance image demonstrating nodular enhancement within the left basal ganglia and left temporal lobe with focal necrosis within the temporal lobe lesion (left). T2-weighted axial magnetic resonance image demonstrating extensive white matter edema within the basal ganglia surrounding a nodular lesion, which is relatively hypointense to edema (right)

CNS disease caused by $M$ tuberculosis is well described in the literature and includes diffuse exudative leptomeningitis, serous tuberculous meningitis, epidural or subdural abscess, and intracerebral or intraspinal tuberculoma. Tuberculomas are most frequently encountered in the cerebrum, but those located in the cerebellum are also described and are most common in children. Tuberculomas are usually $2 \mathrm{~mm}$ to $12 \mathrm{~mm}$ in size, with lobular architecture and commonly central caseous necrosis. The treatment of CNS disease due to $M$ tuberculosis usually involves 12 to 18 months of appropriate therapy.

CNS tuberculomas due to wild-type $M$ bovis have been described in case reports/series $(4,13)$. CNS disease caused by BCG vaccination is extremely rare, with most case reports describing disease only in immunocompromised children (5).

To our knowledge, the present report is the first to describe a case of $\mathrm{M}$ bovis $\mathrm{BCG}$ causing a CNS tuberculoma in an otherwise immunocompetent patient without any evidence of systemic dissemination after intravesical therapy for bladder cancer. Our patient had received multiple instillations of BCG over a four-year period, the most recent one occurring approximately three years before presentation. Isoniazid resistance was concerning, although we could not ascertain the batch or the strain that the patient received. Isoniazid-resistant BCG lymphadenitis has been rarely described in case reports $(14,15)$.

Our patient responded well to a combination therapy with rifampin, ethambutol and moxifloxacin. A recent study (16) showed that moxifloxacin penetrates well into the CSF. Adequate CSF levels of the fluoroquinolone were recorded in all patients who were involved. Of note, rifampin decreases moxifloxacin concentrations, and dose adjustment may be considered in patients receiving both medications. $(16,17)$.

Our case illustrates a rare, but serious infectious complication of intravesical BCG therapy for bladder cancer. Patients undergoing this therapy should be closely monitored for development of these complications. Prolonged antitubercular therapy, possibly including moxifloxacin, appears to be beneficial in the treatment of CNS tuberculous infections.

\section{REFERENCES}

1. Morales A, Eidinger D, Bruce AW. Intracavitary Bacille Calmette-Guérin in the treatment of superficial bladder tumors. J Urol 1976;116:180-3.

2. Lamm DL. Efficacy and safety of Bacille Calmette-Guérin immunotherapy in superficial bladder cancer. Clin Infect Dis 2000;31(Suppl 3):S86-90.

3. Gonzalez OY, Musher DM, Brar I, et al. Spectrum of Bacille Calmette-Guérin (BCG) infection after intravesical BCG immunotherapy. Clin Infect Dis 2003;36:140-8. 
4. Labhard N, Nicod L, Zellweger JP. Cerebral tuberculosis in the immunocompetent host: 8 cases observed in Switzerland. Tuber Lung Dis 1994;75:454-9.

5. Coppes MJ, Olivieri NF, Howes M, Pusic M, Gold R, Richardson SE. Mycobacterial brain abscess possibly due to BCG in an immunocompromised child. Clin Infect Dis 1992;14:662-5.

6. Pedersen FK, Engbaek HC, Hertz H, Vergmann B. Fatal BCG infection in an immunocompetent girl. Acta Pediatr Scand 1978;67:519-23.

7. Parsons LM, Brosch R, Cole ST, et al. Rapid and simple approach for identification of Mycobacterium tuberculosis complex isolates by PCR-based genomic deletion analysis. J Clin Microbiol 2002;40:2339-45.

8. Robert J, Boulahbal F, Trystram D, et al. A national survey of human Mycobacterium bovis infection in France. Int J Tuberc Lung Dis 1999;3:711-4.

9. Hlavsa MC, Moonan PK, Cowan LS, et al. Human tuberculosis due to Mycobacterium bovis in the United States, 1995-2005. Clin Infect Dis 2008;47:168-75.

10. Prescott S, Jackson AM, Hawkyard SJ, Alexandroff AB, James K. Mechanisms of action of intravesical Bacille Calmette-Guérin: Local immune mechanisms. Clin Infect Dis 2000;31(Suppl 3):S91-3.
11. Shelley MD, Court JB, Kynaston H, Wilt TJ, Fish RG, Mason M. Intravesical Bacillus Calmette-Guérin in Ta and T1 Bladder Cancer. Cochrane Database Syst Rev 2000;(4):CD001986.

12. Lamm DL. Complications of bacillus Calmette-Guérin immunotherapy. Urol Clin North Am 1992;19:565-72.

13. Guest SS, Sivit CJ, Meisler WJ, Stevens AC, Simon GL. Intracranial tuberculosis due to Mycobacterium bovis. Comput Radiol 1987;11:151-4.

14. Hesseling AC, Schaaf HS, Victor T, et al. Resistant Mycobacterium bovis BCG disease: Implications for management of BCG disease in HIV-infected children. Pediatr Infect Dis J 2004;23:476-9.

15. Barouni AS, Augusto C, Queiroz MV, Lopes MT, Zanini MS, Salas CE. BCG lymphadenopathy detected in a BCG-vaccinated infant. Braz J Med Biol Res 2004;37:697-700.

16. Alffenaar JW, van Altena R, Bökkerink HJ, et al. Pharmacokinetics of moxifloxacin in cerebrospinal fluid and plasma in patients with tuberculous meningitis. Clin Infect Dis 2009;49:1080-2.

17. Nijland HM, Ruslami R, Suroto AJ, et al. Rifampicin reduces plasma concentrations of moxifloxacin in patients with tuberculosis. Clin Infect Dis 2007;45:1001-7. 


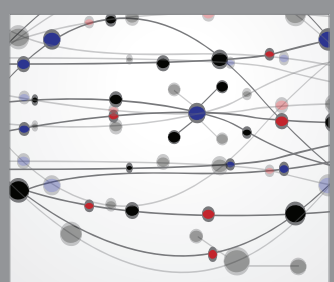

The Scientific World Journal
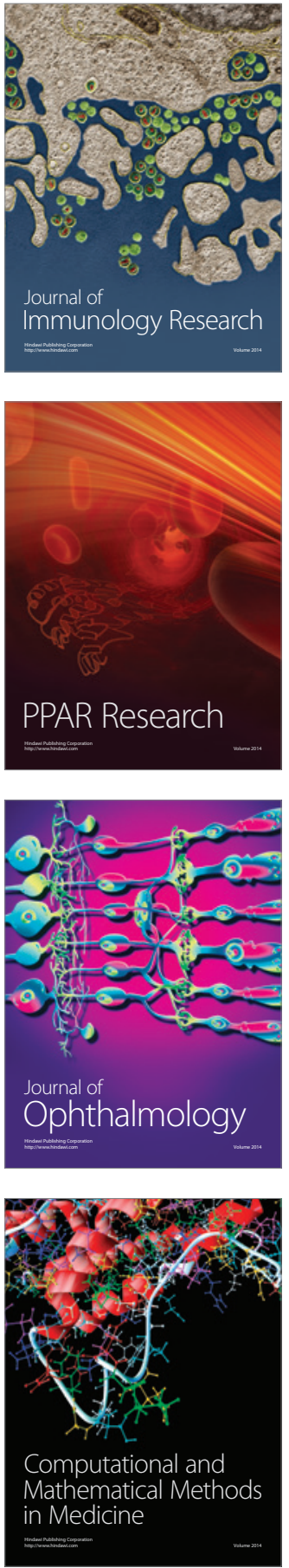

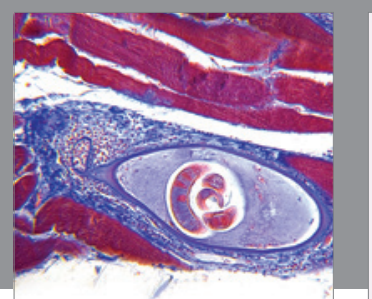

Gastroenterology Research and Practice

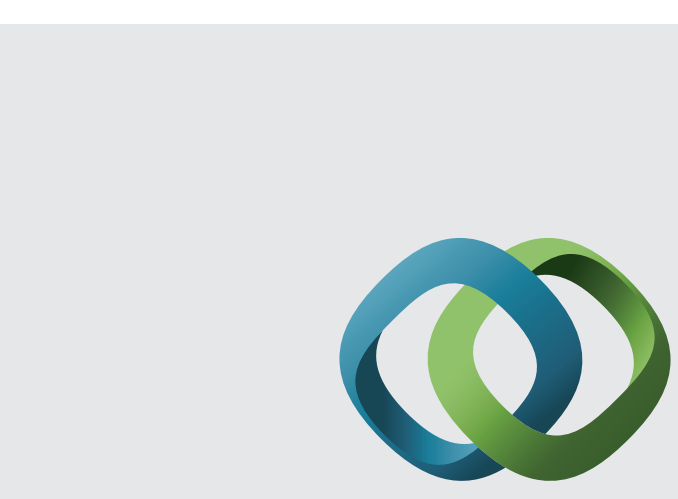

\section{Hindawi}

Submit your manuscripts at

http://www.hindawi.com
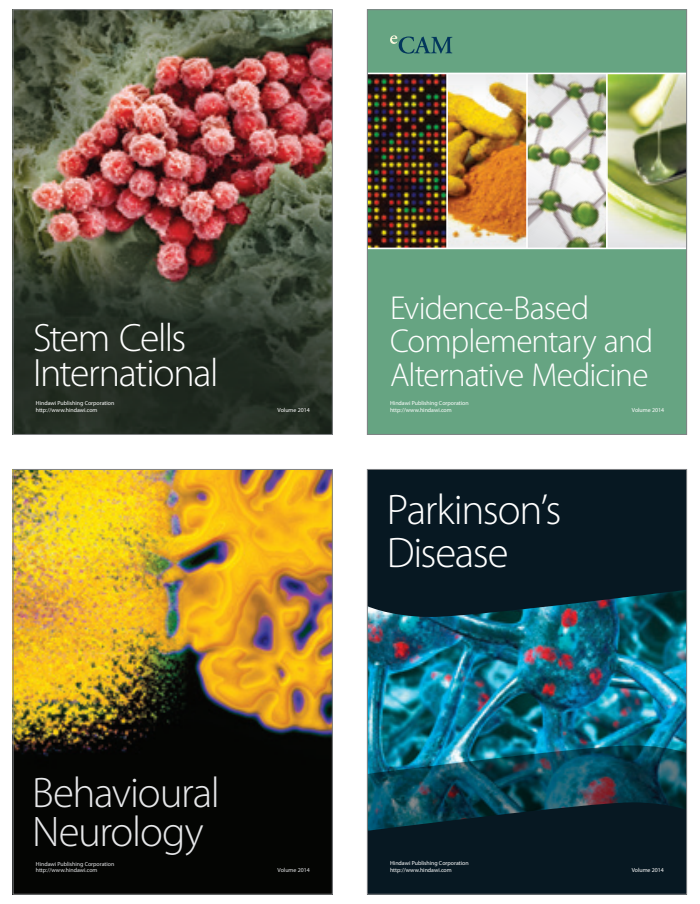
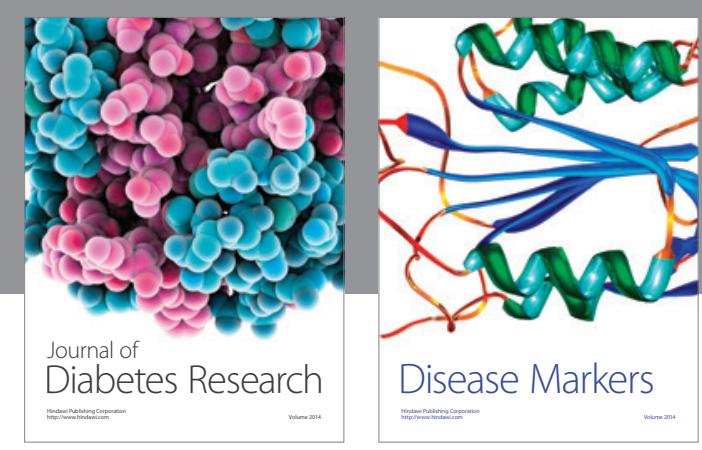

Disease Markers
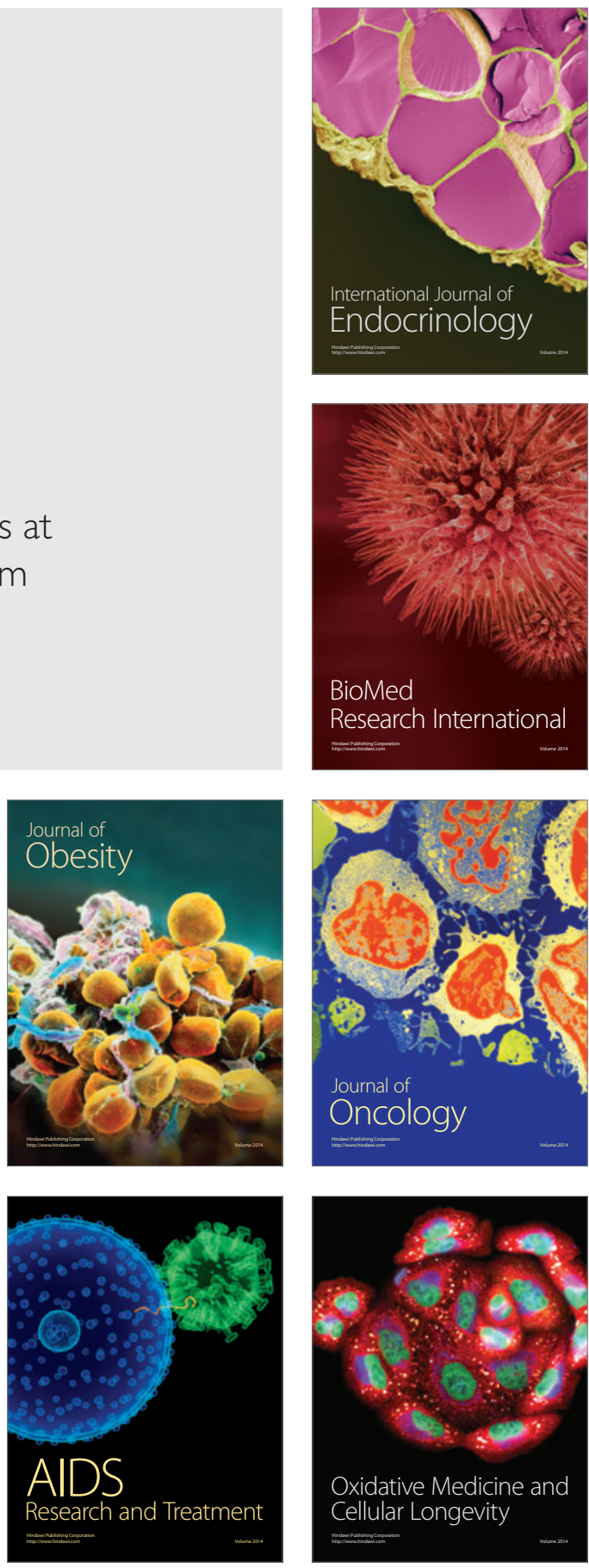